\title{
A Geometric View of Interest Rate Theory *
}

\author{
Tomas Björk ${ }^{\dagger}$ \\ Department of Finance \\ Stockholm School of Economics \\ Box 6501 \\ S-113 83 Stockholm SWEDEN \\ e-mail: tomas.bjork@hhs.se
}

August 11, 2000

To appear in

Handbook of Mathematical Finance, Cambridge University Press

\begin{abstract}
The purpose of this essay is to give an overview of some recent work concerning structural properties of the evolution of the forward rate curve in an arbitrage free bond market. The main problems to be discussed are as follows.

- When is a given forward rate model consistent with a given family of forward rate curves?

- When can the inherently infinite dimensional forward rate process be realized by means of a finite dimensional state space model.

We consider interest rate models of Heath-Jarrow-Morton type, where the forward rates are driven by a multidimensional Wiener process, and where he volatility is allowed to be an arbitrary smooth functional of the present forward rate curve. Within this framwork we give necessary and sufficient conditions for consistency, as well as for the existence of a finite dimensional realization, in terms of the forward rate volatilities.
\end{abstract}

* Support from ITM is gratefully acknowledged.

${ }^{\dagger}$ I am grateful to D. Filipovic and J. Zabzcyk for a number of very helpful discussions. A number of valuable comments from an unknown referee on one of the underlying papers helped to improve that part considerably. I am also highly indebted to B.J. Christensen, A. Gombani and L. Svensson for their generosity in letting me use our joint results for this overview. 


\section{Contents}

1 Introduction $\quad 3$

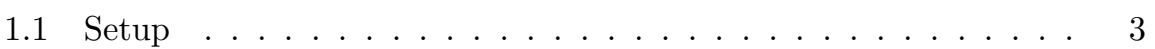

1.2 Main Problems . . . . . . . . . . . . . . . . 4

2 Linear Realization Theory 4

2.1 Deterministic Forward Rate Volatilities . . . . . . . . . . 5

2.2 Existence of Finite Linear Realizations . . . . . . . . . . . . . . . 7

2.3 Transfer Functions . . . . . . . . . . . . . . . . . 8

2.4 Minimal Realizations . . . . . . . . . . . . . . 11

2.5 Economic Interpretation of the State Space . . . . . . . . . . 12

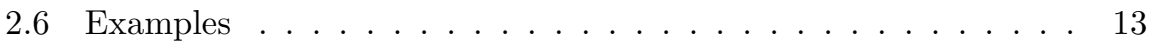

2.7 Notes . . . . . . . . . . . . . . . . . . . 15

3 Invariant Manifolds $\quad 16$

3.1 Parameter Recalibration . . . . . . . . . . . . . . . 16

3.2 Invariant Manifolds . . . . . . . . . . . . . . . . . . . 18

3.3 The Formalized Problem . . . . . . . . . . . . . . . . . 19

3.3.1 The Space . . . . . . . . . . . . . . . . 19

3.3.2 The Forward Curve Manifold . . . . . . . . . . . . . . . . 19

3.3 .3 The Interest Rate Model . . . . . . . . . . . . . . . . 20

3.3.4 The Problem .................. 20

3.4 The Invariance Conditions . . . . . . . . . . . . . . . . 20

3.5 Examples ..................... . . 23

3.5.1 The Nelson-Siegel Family . . . . . . . . . . . . . . 23

3.5.2 The Hull-White and Ho-Lee Models . . . . . . . . . . . . 24

3.6 Notes ....................... . . 25

4 Existence of Nonlinear Realizations $\quad 26$

4.1 Setup ........................ 26

4.2 The Geometric problem . . . . . . . . . . . . . . 27

4.3 The Main Result . . . . . . . . . . . . . . . . . . . . 28 
4.4 Applications . . . . . . . . . . . . . . . . . . . 31

4.4 .1 Constant Volatility . . . . . . . . . . . . . . . . 31

4.4.2 Constant Direction Volatility . . . . . . . . . . . . 32

4.4.3 When is the Short Rate a Markov Process? . . . . . . . . 34

4.5 Notes . . . . . . . . . . . . . . . . . . . . 36

\section{Introduction}

\section{$1.1 \quad$ Setup}

We consider a bond market model (see [2], [27]) living on a filtered probability space $(\Omega, \mathcal{F}, \mathbf{F}, Q)$ where $\mathbf{F}=\left\{\mathcal{F}_{t}\right\}_{t>0}$. The basis is assumed to carry a standard $m$-dimensional Wiener process $W$, and we also assume that the filtration $\mathbf{F}$ is the internal one generated by $W$.

By $p(t, x)$ we denote the price, at $t$, of a zero coupon bond maturing at $t+x$, and the forward rates $r(t, x)$ are defined by

$$
r(t, x)=-\frac{\partial \log p(t, x)}{\partial x} .
$$

Note that we use the Musiela parameterisation, where $x$ denotes the time to maturity. The short rate $R$ is defined as $R(t)=r(t, 0)$, and the money account $B$ is given by $B(t)=\exp \left\{\int_{0}^{t} R(s) d s\right\}$. The model is assumed to be free of arbitrage in the sense that the measure $Q$ above is a martingale measure for the model. In other words, for every fixed time of maturity $T \geq 0$, the process $Z(t, T)=p(t, T-t) / B(t)$ is a $Q$-martingale.

Let us now consider a given forward rate model of the form

$$
\left\{\begin{aligned}
d r(t, x) & =\beta(t, x) d t+\sigma(t, x) d W, \\
r(0, x) & =r^{o}(0, x) .
\end{aligned}\right.
$$

where, for each $x, \beta$ and $\sigma$ are given optional processes. The initial curve $\left\{r^{o}(0, x) ; x \geq 0\right\}$ is taken as given. It is interpreted as the observed forward rate curve.

The standard Heath-Jarrow-Morton drift condition ([20]) can easily be transferred to the Musiela parameterisation. The result (see [6], [26]) is as follows.

Proposition 1.1 (The Forward Rate Equation) Under the martingale measure $Q$ the $r$-dynamics are given by

$$
\begin{aligned}
d r(t, x) & =\left\{\frac{\partial}{\partial x} r(t, x)+\sigma(t, x) \int_{0}^{x} \sigma(t, u)^{\star} d u\right\} d t+\sigma(t, x) d W(t), \\
r(0, x) & =r^{o}(0, x) .
\end{aligned}
$$


where ${ }^{\star}$ denotes transpose.

\subsection{Main Problems}

Suppose now that we are give a concrete model $\mathcal{M}$ within the above framework, i.e. suppose that we are given a concrete specification of the volatility process $\sigma$. We now formulate a couple of natural problems:

1. Take, in addition to $\mathcal{M}$, also as given a parameterized family $\mathcal{G}$ of forward rate curves. Under which conditions is the family $\mathcal{G}$ consistent with the dynamics of $\mathcal{M}$ ? Here consistency is interpreted in the sense that, given an initial forward rate curve in $\mathcal{G}$, the interest rate model $\mathcal{M}$ will only produce forward rate curves belonging to the given family $\mathcal{G}$.

2. When can the given, inherently infinite dimensional, interest rate model $\mathcal{M}$ be written as a finite dimensional state space model? More precisely, we seek conditions under which the forward rate process $r(t, x)$ induced by the model $\mathcal{M}$, can be realized by a system of the form

$$
\begin{aligned}
d Z_{t} & =a\left(Z_{t}\right) d t+b\left(Z_{t}\right) d W_{t}, \\
r(t, x) & =G\left(Z_{t}, x\right) .
\end{aligned}
$$

where $Z$ (interpreted as the state vector process) is a finite dimensional diffusion, $a(z), b(z)$ and $G(z, x)$ are deterministic functions and $W$ is the same Wiener process as in in (2).

As will be seen below, these two problems are intimately connected, and the main purpose of this chapter is to give an overview of some recent work in this area. The text is mainly based on [3], [4] and [5], but the presentation given below is more focused on geometric intuition than the original articles, where full proofs, technical details and further results can be found. In the analysis below we use ideas from systems and control theory (see [24]) as well as from nonlinear filtering theory (see [8]). References to the literature will sometimes be given in the text, but will mainly be summarised in the Notes at the end of each section.

The organisation of the text is as follows. In Section 2 we study the existence of a finite dimensional factor realization in the comparatively simple case when the forward rate volatilities are deterministic. In Section 3 we study the general consistency problem, and in Section 4 we use the consistency results from Section 3 in order to give a fairly complete picture of the nonlinear realization problem.

\section{Linear Realization Theory}

In the general case, the forward rate equation (2) is a highly nonlinear infinite dimensional SDE but, as can be expected, the special case of linear dynamics is 
much easier to handle. In this section we therefore concentrate on linear forward rate models, and look for finite dimensional linear realizations.

\subsection{Deterministic Forward Rate Volatilities}

For the rest of the section we only consider the case when the volatility $\sigma(t, x)=$ $\left[\sigma_{1}(t, x), \ldots, \sigma_{m}(t, x)\right]$ is a deterministic time-independent function $\sigma(x)$ of $x$ only.

Assumption 2.1 The volatility $\sigma$ is a deterministic $C^{\infty}{ }_{-}$mapping $\sigma: R_{+} \rightarrow$ $R^{m}$

Denoting the function $x \longmapsto r(t, x)$ by $r(t)$ we have, from (2),

$$
\begin{aligned}
d r(t) & =\{\mathbf{F} r(t)+D\} d t+\sigma d W(t), \\
r(0) & =r^{o}(0) .
\end{aligned}
$$

Here the linear operator $\mathbf{F}$ is defined by

$$
\mathbf{F}=\frac{\partial}{\partial x},
$$

whereas the function $D$ is given by

$$
D(x)=\sigma(x) \int_{0}^{x} \sigma(s)^{\star} d s .
$$

The point to note here is that, because of our choice of a deterministic volatility $\sigma(x)$, the forward rate equation (6) is a linear (or rather affine) SDE. Because of this linearity (albeit in infinite dimensions) we therefore expect to be able to provide an explicit solution of (6). We now recall that a scalar equation of the form

$$
d y(t)=[a y(t)+b] d t+c d W(t)
$$

has the solution

$$
y(t)=e^{a t} y(0)+\int_{0}^{t} e^{a(t-s)} b d s+\int_{0}^{t} e^{a(t-s)} c d W(s),
$$

and we are led to conjecture that the solution to (6) is given by the formal expression

$$
r(t)=e^{\mathbf{F} t} r^{o}+\int_{0}^{t} e^{\mathbf{F}(t-s)} D d s+\int_{0}^{t} e^{\mathbf{F}(t-s)} \sigma d W(s) .
$$

The formal exponential $e^{\mathbf{F} t}$ acts on real valued functions, and we have to figure out how it operates. From the standard series expansion of the exponential function one is led to write

$$
\left[e^{\mathbf{F} t} f\right](x)=\sum_{n=0}^{\infty} \frac{t^{n}}{n !}\left[F^{n} f\right](x)
$$


In our case $F^{n}=\frac{\partial^{n}}{\partial x^{n}}$, so (assuming $f$ to be analytic) we have

$$
\left[e^{\mathbf{F} t} f\right](x)=\sum_{n=0}^{\infty} \frac{t^{n}}{n !} \frac{\partial^{n} f}{\partial x^{n}}(x)
$$

This is, however, just a Taylor series expansion of $f$ around the point $x$, so for analytic $f$ we have $\left[e^{\mathbf{F} t} f\right](x)=f(x+t)$. We have in fact the following precise result (which can be proved rigorously).

Proposition 2.1 The operator $\mathbf{F}$ is the infinitesimal generator of the semigroup of left translations, i.e. for any $f \in C[0, \infty)$ we have

$$
\left[e^{\mathbf{F} t} f\right](x)=f(t+x)
$$

The solution of the forward rate equation (6) is given by as

$$
r(t, x)=e^{\mathbf{F} t} r^{o}(0, x)+\int_{0}^{t} e^{\mathbf{F}(t-s)} D(x) d s+\int_{0}^{t} e^{\mathbf{F}(t-s)} \sigma(x) d W(s)
$$

or equivalently by

$$
r(t, x)=r^{o}(0, x+t)+\int_{0}^{t} D(x+t-s) d s+\int_{0}^{t} \sigma(x+t-s) d W(s) .
$$

From (12) it is clear by inspection that we may write the forward rate equation (6) as

$$
\begin{aligned}
d r_{0}(t, x) & =\mathbf{F} r_{0}(t, x) d t+\sigma(x) d W(t), \quad r_{0}(0, x)=0 \\
r(t, x) & =r_{0}(t, x)+\delta(t, x)
\end{aligned}
$$

where $\delta$ is given by

$$
\delta(t, x)=r^{o}(0, x+t)+\int_{0}^{t} D(x+t-s) d s
$$

Since $\delta(t, x)$ is not affected by the input $W$, we see that the problem of finding a realization for the term structure system (6) is equivalent to that of finding a realization for (14). We are thus led to the following definition.

Definition 2.1 A matrix triple $[A, B, C(x)]$ is called an $n$-dimensional realization of the systems (6) and (14) if $r_{0}$ has the representation

$$
\begin{aligned}
d Z(t) & =A Z(t) d t+B d W(t), \quad Z(0)=0 . \\
r_{0}(t, x) & =C(x) Z(t)
\end{aligned}
$$


Our main problems are now as follows.

- Take as a priori given a volatility structure $\sigma(x)$.

- When does there exists a finite dimensional realization?

- If there exists a finite dimensional realization, what is the minimal dimension?

- How do we construct a minimal realization from knowledge of $\sigma$ ?

- Is there an economic interpretation of the state process $Z$ in the realization?

\subsection{Existence of Finite Linear Realizations}

We will now go on to study the existence of a finite dimensional realization of the stochastic system (14), and in order to get some ideas, suppose that there actually exists a finite dimensional realisation of (14) of the form (17)-(18). Solving (14) we have

$$
r_{0}(t, x)=\int_{0}^{t} e^{\mathbf{F}(t-s)} \sigma(x) d W(s)=\int_{0}^{t} \sigma(x+t-s) d W(s),
$$

while, from the realization (17)-(18), we also have

$$
r_{0}(t, x)=C(x) Z(t)=C(x) \int_{0}^{t} e^{A(t-s)} B d W(s)
$$

Thus we have, with probability one, for each $x$ and each $t$,

$$
\int_{0}^{t} \sigma_{x}(t-s) d W(s)=\int_{0}^{t} C(x) e^{A(t-s)} B d W(s)
$$

where we use subindex $x$ to denote left translation, i.e. $f_{x}(t)=f(x+t)$. This leads us immediately to conjecture that the equation

$$
\sigma_{x}(t)=C(x) e^{A t} B
$$

must hold for all $x$ and $t$, and we have our first main result.

\section{Proposition 2.2}

1. The forward rate process has a finite dimensional linear realization if and only if the volatility function $\sigma$ can be written on the form

$$
\sigma(x)=C_{0} e^{A x} B
$$


2. If $\sigma$ has the form (20) then a concrete realization of $r_{0}$ is given by

$$
\begin{aligned}
d Z(t) & =A Z(t) d t+B d W(t), \quad Z(0)=0 . \\
r_{0}(t, x) & =C(x) Z(t),
\end{aligned}
$$

with $A, B$ as in (20), and with $C(x)=C_{0} e^{A x}$. The forward rates $r(t, x)$ are then given by (15)-(16).

Proof. It is clear from the discussion above that if there exists a finite realisation, then we must have the factorisation $\sigma_{x}(t)=C(x) e^{A t} B$. Setting $x=0$, and denoting $C(0)$ by $C_{0}$, in this gives us the relation (20). If, on the other hand, $\sigma$ factors as in (20), then we simply define $Z$ as in (21). A direct calculation as above then shows that we have $r_{0}(t, x)=C_{0} e^{A x} z(t)$.

Remark 2.1 Let us call a function of the form $c e^{A x} b$, where $c$ is a row vector, $A$ is a square matrix and $b$ is a column vector, a quasi-exponential (or $\mathrm{QE}$ ) function. The general form of a quasi-exponential function $f$ is given by

$$
f(x)=\sum_{i} e^{\lambda_{i} x}+\sum_{j} e^{\alpha_{i} x}\left[p_{j}(x) \cos \left(\omega_{j} x\right)+q_{j}(x) \sin \left(\omega_{j} x\right)\right],
$$

where $\lambda_{i}, \alpha_{1}, \omega_{j}$ are real numbers, whereas $p_{j}$ and $q_{j}$ are real polynomials.

QE functions will turn up again, so we list some simple properties.

Lemma 2.1 The following hold for the quasi-exponential functions.

- A function is $Q E$ if and only if it is a component of the solution of a vector valued linear ODE with constant coefficients.

- A function is $Q E$ if and only if it can be written as $f(x)=c e^{A x} b$.

- If $f$ is $Q E$, then $f^{\prime}$ is $Q E$.

- If $f$ is $Q E$, then its primitive function is $Q E$.

- If $f$ and $g$ are $Q E$, then $f g$ is $Q E$.

\subsection{Transfer Functions}

Using ideas from linear systems theory, an alternative view of the realization problem is obtained by studying transfer functions, i.e. by going to the frequency domain. To get some intuition, consider again the equation

$$
d r_{0}(t, x)=\mathbf{F} r_{0}(t, x) d t+\sigma(x) d W(t), \quad r_{0}(0, x)=0 .
$$


Let us now formally "divide by $d t$ ", which gives us

$$
\frac{d r_{0}}{d t}(t, x)=\mathbf{F} r_{0}(t, x)+\sigma(x) \frac{d W}{d t}(t),
$$

where the formal time derivative $\frac{d W}{d t}(t)$ is interpreted as white noise. We interpret this equation as an input-output system where the random input signal $t \longmapsto \frac{d W}{d t}(t)$ is transformed into the infinite dimensional output signal $t \longmapsto r_{0}(t, \cdot)$. We thus view the equation as a version of the following controlled ODE

$$
\begin{aligned}
\frac{d r_{0}}{d t}(t, x) & =\mathbf{F} r_{0}(t, x)+\sigma(x) u(t), \\
r_{0}(0) & =0,
\end{aligned}
$$

where $u$ is a deterministic input signal. Generally speaking, tricks like this does not work directly, since we are ignoring the difference between standard differential calculus, which is used to analyze (25), and Itô calculus which we use when dealing with SDEs. In this case, however, because of the linear structure, the second order Itô term will not come into play, so we are safe. (See the discussion in Section 3.4 around the Stratonovich integral for how to treat the nonlinear situation.)

It is now natural to study the transfer function for the system (25), which relates the Laplace transform of the input signal to the Laplace transform of the output signal.

Definition 2.2 The transfer function, $K(s, x)$, for (25) is determined by the relation

$$
\tilde{r}_{0}(s, x)=K(s, x) \tilde{u}(s),
$$

where $\sim$ denotes the Laplace transform in the t-variable.

From the uniqueness of the Laplace transform we then have the following result.

Lemma 2.2 The system

$$
\begin{aligned}
d Z(t) & =A Z(t) d t+B d W(t), \quad Z(0)=0 . \\
r_{0}(t, x) & =C(x) Z(t),
\end{aligned}
$$

is a realization of

$$
d r_{0}(t, x)=\mathbf{F} r_{0}(t, x) d t+\sigma(x) d W(t), \quad r_{0}(0, x)=0
$$

if and only if the deterministic control system

$$
\frac{d r_{0}}{d t}(t, x)=\mathbf{F} r_{0}(t, x)+\sigma(x) u(t),
$$


has the same transfer function as the system

$$
\begin{aligned}
\frac{d Z}{d t}(t) & =A Z(t)+B u(t), \\
r_{0}(t, x) & =C(x) Z(t) .
\end{aligned}
$$

Furthermore we have

Lemma 2.3 The transfer function $K(s, x)$ of (29) is given by

$$
K(s, x)=\mathcal{L}\left[\sigma_{x}\right](s),
$$

where $\mathcal{L}$ denotes the Laplace transform, and $\sigma_{x}$ denotes left translation.

Proof. From (29) we have

$$
r_{0}(t, x)=\int_{0}^{t} \sigma(x+t-s) u(s) d s=\left[\sigma_{x} \star u\right](t),
$$

and thus

$$
\tilde{r}_{0}(s, x)=\mathcal{L}\left[\sigma_{x}\right](s) \tilde{u}(s)
$$

For concrete computation of a realization, the following result is useful.

\section{Lemma 2.4}

- The transfer function of the system (30)-(31) is given by

$$
K(s, x)=C(x)[s I-A]^{-1} B .
$$

- The $r_{0}$ system has a finite realization if and only if there exists a factorization of the form

$$
\mathcal{L}\left[\sigma_{x}\right](s)=C(x)[s I-A]^{-1} B
$$

- Denote the transfer function of $r_{0}$ by $K(s, x)$, and assume that that there exits a finite dimensional realization. If we have fund $A, B$ and $C$ such that

$$
K(s, 0)=C[s I-A]^{-1} B,
$$

then a realization of $r_{0}$ is given by $\left[A, B, C e^{A x}\right]$. 
Proof. The first assertion is immediately obtained by taking the Laplace transform of (30)-(31). The second follows at from Lemma 2.2, and the third from Proposition 2.2.

If we want to find a concrete realization for a given system, we thus have two possibilities. We can either look for a factorization of the volatility function as $\sigma(x)=C e^{A x} B$, or we can try to factor the transfer function as $K(s, 0)=$ $C[s I-A]^{-1} B$. From a logical point of view the two approaches are equivalent, but from a practical point of view it is much easier to factor the transfer function than to factor the volatility. There are in fact a number of standard algorithms in the systems theoretic literature which construct a realization, given knowledge of the transfer functions. See [7].

\subsection{Minimal Realizations}

The purpose of this section is to determine the minimal dimension of a finite dimensional realization.

Definition 2.3 The dimension of a realization $[A, B, C(x)]$ is defined as the dimension of the corresponding state space. A realization $[A, B, C(x)]$ is said to be minimal if there is no other realization with smaller dimension. The McMillan-degree, $\mathcal{D}$, of the forward rate system is defined as the dimension of a minimal realization.

In order to get a feeling for how to determine the McMillan degree, we note that $r_{0}$ has a finite dimensional realization if and only if $r_{0}$ evolves on a finite dimensional subspace in the infinite dimensional function space $\mathcal{H}$. Furthermore, it seems obvious that the McMillan degree equals the dimension of this subspace.

In order to determine the subspace above, let us again view the $r_{0}$ system as a special case of the following controlled equation, where we have suppressed $x$.

$$
\left\{\begin{aligned}
\frac{d r_{0}}{d t} & =\mathbf{F} r_{0}(t)+\sigma u(t), \\
r_{0}(0) & =0 .
\end{aligned}\right.
$$

The solution of this equation is given by

$$
r_{0}(t)=\int_{0}^{t} e^{\mathbf{F}(t-s)} \sigma u(s) d s=\int_{0}^{t} \sum_{0}^{\infty} \frac{(t-s)^{n}}{n !} \mathbf{F}^{n} \sigma u(s) d s .
$$

This is a linear combination of vectors of the form $\mathbf{F}^{n} \sigma_{i}$, so we see that the smallest subspace $\mathcal{R}$ which contains $r_{0}(t)$ for all $t$ and for all choices of the input signal $u$ is given by

$$
\mathcal{R}=\operatorname{span}\left[\sigma, \mathbf{F} \sigma, \mathbf{F}^{2} \sigma, \cdots\right]=\operatorname{span}\left[\mathbf{F}^{k} \sigma_{i} ; i=1, \cdots, m . k=0,1, \cdots\right]
$$

We thus have the following result. 
Proposition 2.3 Take the volatility function

$$
\sigma=\left[\sigma_{1}, \cdots, \sigma_{m}\right]
$$

as given. Then the McMillan degree, $\mathcal{D}$, is given by

$$
\mathcal{D}=\operatorname{dim}(\mathcal{R}),
$$

with $\mathcal{R}$ defined as in (34). The forward rate system thus admits a finite dimensional realization if and only if the space spanned by the components of $\sigma$ and all their derivatives is finite dimensional.

\subsection{Economic Interpretation of the State Space}

In general, the state space of the minimal realization of a given system has no concrete (e.g. physical) interpretation. In our case, however, the states of the minimal realization turn out to have a simple economic interpretation in terms of a minimal set of "benchmark" forward rates.

Assume that $[A, B, C]$ is a minimal realization, of dimension $n$, of the forward rates as in (21)-(22). Let us choose a set of "benchmark" maturities $x_{1}, \cdots, x_{n}$. We use the notation $\bar{x}=\left(x_{1}, \cdots, x_{n}\right)$. Assume furthermore that the maturity vector $\bar{x}$ is chosen so that the matrix

$$
T(\bar{x})=\left[\begin{array}{c}
C e^{A x_{1}} \\
\vdots \\
C e^{A x_{n}}
\end{array}\right]
$$

is invertible. It can be shown (see [4]) that, outside a set of measure zero, this can always be done be done as long as the maturities are distinct. We use the notation

$$
r_{0}(t, \bar{x})=\left[\begin{array}{c}
r_{0}\left(t, x_{1}\right) \\
\vdots \\
r_{0}\left(t, x_{n}\right)
\end{array}\right]
$$

and corresponding interpretations for column vectors like $r(t, \bar{x}), \delta(t, \bar{x})$ etc.

The following result shows how the entire term structure is determined by the benchmark forward rates.

Proposition 2.4 Assume that (21)-(22) is a minimal realization of the forward rates, and assume furthermore that a maturity vector $\bar{x}=\left(x_{1}, \cdots, x_{n}\right)$ is chosen as above. Then the following hold.

- With notation as above, the vector $r(t, \bar{x})$ of benchmark forward rates has the dynamics

$$
\begin{aligned}
d r(t, \bar{x}) & =\left[T(\bar{x}) A T^{-1}(\bar{x}) r(t, \bar{x})+\Psi(t, \bar{x})\right] d t+T(\bar{x}) B d W(t) \\
r(0, \bar{x}) & =r^{\star}(0, \bar{x})
\end{aligned}
$$


where the deterministic function $\Psi$ is given by

$$
\Psi(t, \bar{x})=\frac{\partial r^{\star}}{\partial x}(0, t \bar{e}+\bar{x})+D(t \bar{e}+\bar{x})-T(\bar{x}) A T^{-1}(\bar{x}) \delta(t, \bar{x})
$$

Here $\bar{e} \in R^{n}$ denotes the vector with unit components, i.e.

$$
\bar{e}=\left[\begin{array}{c}
1, \\
1, \\
\vdots \\
1
\end{array}\right]
$$

- The system of benchmark forward rates determine the entire forward rate process according to the formula

$$
r(t, x)=C e^{A x} T^{-1}(\bar{x}) r(t, \bar{x})-C e^{A x} T^{-1}(\bar{x}) \delta(t, \bar{x})+\delta(t, x) .
$$

- The correspondence between $Z$ and $r$ is given by

$$
r_{0}(t, \bar{x})=T(\bar{x}) Z(t)
$$

Proof. See [4].

The conclusion is thus that the state variables of a minimal realization can be interpreted as an affine transformation of a vector of benchmark forward rates.

\subsection{Examples}

In this section we will give some simple illustrations of the theory. Note the handling of multiple roots of the matrix $A$, and the fact that the input noise can have dimension smaller than the dimension of $A$.

Example 2.1 $\sigma(x)=\sigma e^{-a x}$

We consider a model driven by a one dimensional Wiener process, having the forward rate volatility structure

$$
\sigma(x)=\sigma e^{-a x}
$$

where $\sigma$ in the right hand side denotes a constant. (The reader will probably recognize this example as the Hull-White model.) We start by determining the McMillan degree $\mathcal{D}$, and by Proposition 2.3 we have

$$
\mathcal{D}=\operatorname{dim}(\mathcal{R})
$$


where the space $\mathcal{R}$ is given by

$$
\mathcal{R}=\operatorname{span}\left[\frac{d^{k}}{d x^{k}} \sigma e^{-a x} ; k \geq 0\right] .
$$

It is obvious that $\mathcal{R}$ is one dimensional, and that it is spanned by the single function $e^{-a x}$. Thus the McMillan degree is given by $\mathcal{D}=1$. We now want to apply Proposition 2.2 to find a realization, so we must factor the volatility function. In this case this is easy, since we have the trivial factorization $\sigma(x)=$ $1 \cdot e^{-a x} \cdot \sigma$. In the notation of Proposition 2.2 we thus have

$$
\begin{aligned}
C_{0} & =1, \\
A & =-a, \\
B & =\sigma .
\end{aligned}
$$

A realization of the forward rates is thus given by

$$
\begin{aligned}
d Z(t) & =-a Z(t) d t+\sigma d W(t), \\
r_{0}(t, x) & =e^{-a x} Z(t), \\
r(t, x) & =r_{0}(t, x)+\delta(t, x),
\end{aligned}
$$

and since the state space in this realization is of dimension one, the realization is minimal. We see that if $a>0$ then the system is asymptotically stable.

We now go on to the interpretation of the state space, and since $\mathcal{D}=1$ we can choose a single benchmark maturity. The canonical choice is of course $x_{1}=0$, i.e. we choose the instantaneous short rate $R(t)$ as the state variable. In the notation of Proposition 2.4 we then have

$$
\begin{aligned}
T(\bar{x}) & =1, \\
r(t, \bar{x}) & =R(t),
\end{aligned}
$$

and we get rate dynamics

$$
d R(t)=\{\Psi(t, 0)-a R(t)\} d t+\sigma d W(t) .
$$

Thus we see that we have indeed the Hull-White extension of the Vasiček model. Note however that we do not have to choose the benchmark maturity as $x_{1}=0$. We can in fact choose any fixed maturity, $x_{1}$ and then use the corresponding forward rate as benchmark. This will give us the dynamics

$$
d r\left(t, x_{1}\right)=\left\{\Psi\left(t, x_{1}\right)-\operatorname{ar}\left(t, x_{1}\right)\right\} d t+e^{-a x_{1}} d W(t),
$$

and now the entire forward rate curve will be determined by the $x_{1}$-rate according to formula (36).

Example $2.2 \sigma(x)=x e^{-a x}$

In this example we still have a single driving Wiener process, but the volatility function is now "hump-shaped". 
By taking derivatives of $\sigma(x)$ we immediately see, from Proposition 2.3 that $\mathcal{R}$ is given by

$$
\mathcal{R}=\operatorname{span}\left[x e^{-a x}, e^{-a x}\right],
$$

so in this case $\mathcal{D}=2$, and we have a two dimensional minimal state space. In order to obtain a realization we compute the transfer function $K(s, x)$, which is given by Lemma 2.3 as

$$
K(s, x)=\mathcal{L}\left[(x+\cdot) e^{-a(x+\cdot)}\right](s) .
$$

An easy calculation gives us

$$
K(s, x)=\frac{e^{-a x}}{(a+s)^{2}}+\frac{x e^{-a x}}{(a+s)}=\frac{s x e^{-a x}+(1+a x) e^{-a x}}{(a+s)^{2}},
$$

and we now look for a realization of this transfer function (for a fixed $x$ ). The obvious thing to do is to use the standard controllable realization (see [7]), and we obtain

$$
\begin{aligned}
C(x) & =\left[x e^{-a x},(1+a x) e^{-a x}\right] \\
A & =\left[\begin{array}{cc}
-2 a & -a^{2} \\
1 & 0
\end{array}\right], \\
B & =\left[\begin{array}{l}
1 \\
0
\end{array}\right] .
\end{aligned}
$$

Since $\mathcal{D}=2$ and this realization is two-dimensional we have a minimal realization, given by

$$
\begin{aligned}
d Z_{1}(t) & =-2 a Z_{1}(t) d t-a^{2} Z_{2}(t) d t+d W(t), \\
d Z_{2}(t) & =Z_{1}(t) d t \\
r_{0}(t, x) & =x e^{-a x} Z_{1}(t)+(1+a x) e^{-a x} Z_{2}(t) \\
r(t, x) & =r_{0}(t, x)+\delta(t, x) .
\end{aligned}
$$

We have a double eigenvalue of the system matrix $A$ at $\lambda_{1}=-a$, so if $a>0$ the system is asymptotically stable.

\section{$2.7 \quad$ Notes}

This section is mainly based on [4]. The first paper to appear in this area was to our knowledge the preprint [26], where the Musiela parameterization and the space $\mathcal{R}$ is discussed in some detail. See also the closely related and interesting preprints [15] [16] and [32]. Because of the linear structure, the theory above is closely connected to (and in a sense inverse to) the theory of affine term structures developed in [13]. The standard reference on infinite dimensional SDEs is [12], where one also can find a presentation of the connections between control theory and infinite dimensional linear stochastic equations. 


\section{Invariant Manifolds}

In this section we study when a given submanifold of forward rate curves is invariant under the action of a given interest rate model. This problem is of interest from an applied as well as from a theoretical point of view. In particular we will use the results from this section to analyze problems about existence of finite dimensional factor realizations for interest rate models on forward rate form. Invariant manifolds are, however, also of interest in their own right, so we begin by discussing a concrete problem which naturally leads to the invariance concept.

\subsection{Parameter Recalibration}

A standard procedure when dealing with concrete interest rate models on a high frequency (say, daily) basis can be described as follows:

1. At time $t=0$, use market data to fit (calibrate) the model to the observed bond prices.

2. Use the calibrated model to compute prices of various interest rate derivatives.

3. The following day $(t=1)$, repeat the procedure in 1 . above in order to recalibrate the model, etc..

To carry out the calibration in step 1 . above, the analyst typically has to produce a forward rate curve $\left\{r^{o}(0, x) ; x \geq 0\right\}$ from the observed data. However, since only a finite number of bonds actually trade in the market, the data consist of a discrete set of points, and a need to fit a curve to these points arises. This curve-fitting may be done in a variety of ways. One way is to use splines, but also a number of parameterized families of smooth forward rate curves have become popular in applications - the most well-known probably being the Nelson-Siegel (see [28]) family. Once the curve $\left\{r^{o}(0, x) ; x \geq 0\right\}$ has been obtained, the parameters of the interest rate model may be calibrated to this.

Now, from a purely logical point of view, the recalibration procedure in step 3 . above is of course slightly nonsensical: If the interest rate model at hand is an exact picture of reality, then there should be no need to recalibrate. The reason that everyone insists on recalibrating is of course that any model in fact only is an approximate picture of the financial market under consideration, and recalibration allows incorporating newly arrived information in the approximation. Even so, the calibration procedure itself ought to take into account that it will be repeated. It appears that the optimal way to do so would involve a combination of time series and cross-section data, as opposed to the purely cross-sectional curve-fitting, where the information contained in previous curves is discarded in each recalibration. . 
The cross-sectional fitting of a forward curve and the repeated recalibration is thus, in a sense, a pragmatic and somewhat non-theoretical endeavour. Nonetheless, there are some nontrivial theoretical problems to be dealt with in this context, and the problem to be studied in this section concerns the consistency between, on the one hand, the dynamics of a given interest rate model, and, on the other hand, the forward curve family employed.

What, then, is meant by consistency in this context? Assume that a given interest rate model $\mathcal{M}$ (e.g. the Hull-White model) in fact is an exact picture of the financial market. Now consider a particular family $\mathcal{G}$ of forward rate curves (e.g. the Nelson-Siegel family) and assume that the interest rate model is calibrated using this family. We then say that the pair $(\mathcal{M}, \mathcal{G})$ is consistent (or, that $\mathcal{M}$ and $\mathcal{G}$ are consistent) if all forward curves which may be produced by the interest rate model $\mathcal{M}$ are contained within the family $\mathcal{G}$. Otherwise, the pair $(\mathcal{M}, \mathcal{G})$ is inconsistent.

Thus, if $\mathcal{M}$ and $\mathcal{G}$ are consistent, then the interest rate model actually produces forward curves which belong to the relevant family. In contrast, if $\mathcal{M}$ and $\mathcal{G}$ are inconsistent, then the interest rate model will produce forward curves outside the family used in the calibration step, and this will force the analyst to change the model parameters all the time - not because the model is an approximation to reality, but simply because the family does not go well with the model.

Put into more operational terms this can be rephrased as follows.

- Suppose that you are using a fixed interest rate model $\mathcal{M}$. If you want to do recalibration, then your family $\mathcal{G}$ of forward rate curves should be chosen is such a way as to be consistent with the model $\mathcal{M}$.

Note however that the argument also can be run backwards, yielding the following conclusion for empirical work.

- Suppose that a particular forward curve family $\mathcal{G}$ has been observed to provide a good fit, on a day-to-day basis, in a particular bond market. Then this gives you modelling information about the choice of an interest rate model in the sense that you should try to use/construct an interest rate model which is consistent with the family $\mathcal{G}$.

We now have a number of natural problems to study.

I Given an interest rate model $\mathcal{M}$ and a family of forward curves $\mathcal{G}$, what are necessary and sufficient conditions for consistency?

II Take as given a specific family $\mathcal{G}$ of forward curves (e.g. the Nelson-Siegel family). Does there exist any interest rate model $\mathcal{M}$ which is consistent with $\mathcal{G}$ ? 
III Take as given a specific interest rate model $\mathcal{M}$ (e.g. the Hull-White model). Does there exist any finitely parameterized family of forward curves $\mathcal{G}$ which is consistent with $\mathcal{M}$ ?

In this section we will mainly address problem (I) above. Problem II has been studied, for special cases, in [17], [18], whereas Problem III can be shown (see Proposition 4.2) to be equivalent to the problem of finding a finite dimensional factor realization of the model $\mathcal{M}$ and we provide a farily complete solution in Section 4.

\subsection{Invariant Manifolds}

We now move on to give precise mathematical definition of the consistency property discussed above, and this leads us to the concept of an invariant manifold.

Definition 3.1 (Invariant manifold) Take as given the forward rate process dynamics (2). Consider also a fixed family (manifold) of forward rate curves $\mathcal{G}$. We say that $\mathcal{G}$ is locally invariant under the action of $r$ if, for each point $(s, r) \in R_{+} \times \mathcal{G}$, the condition $r_{s} \in \mathcal{G}$ implies that $r_{t} \in \mathcal{G}$, on a time interval with positive length. If $r$ stays forever on $\mathcal{G}$, we say that $\mathcal{G}$ is globally invariant.

The purpose of this section is to characterize invariance in terms of local characteristics of $\mathcal{G}$ and $\mathcal{M}$, and in this context local invariance is the best one can hope for. In order to save space, local invariance will therefore be referred to as invariance.

To get some intuitive feeling for the invariance concepts one can consider the following two-dimensional deterministic system

$$
\begin{aligned}
& \frac{d y_{1}}{d t}=y_{2}, \\
& \frac{d y_{2}}{d t}=-y_{1} .
\end{aligned}
$$

For this system it is obvious that the unit circle $\mathcal{C}=\left\{\left(y_{1}, y_{2}\right): y_{1}^{2}+y_{2}^{2}=1\right\}$ is globally invariant, i.e. if we start the system on $\mathcal{C}$ it will stay forever on $\mathcal{C}$. The 'upper half' of the circle, $\mathcal{C}_{u}=\left\{\left(y_{1}, y_{2}\right): y_{1}^{2}+y_{2}^{2}=1, y_{2}>0\right\}$, is on the other hand only locally invariant, since the system will leave $\mathcal{C}_{u}$ at the point $(1,0)$. This geometric situation is in fact the generic one also for our infinite dimensional stochastic case. The forward rate trajectory will never leave a locally invariant manifold at a point in the relative interior of the manifold. Exit from the manifold can only take place at the relative boundary points. We have no general method for determining whether a locally invariant manifold also is globally invariant or not. Problems of this kind has to be solved separately for each particular case. 


\subsection{The Formalized Problem}

\subsubsection{The Space}

As our basic space of forward rate curves we will use a weighted Sobolev space, where a generic point will be denoted by $r$.

Definition 3.2 Consider a fixed real number $\gamma>0$. The space $\mathcal{H}_{\gamma}$ is defined as the space of all differentiable (in the distributional sense) functions

$$
r: R_{+} \rightarrow R
$$

satisfying the norm condition $\|r\|_{\gamma}<\infty$. Here the norm is defined as

$$
\|r\|_{\gamma}^{2}=\int_{0}^{\infty} r^{2}(x) e^{-\gamma x} d x+\int_{0}^{\infty}\left(\frac{d r}{d x}(x)\right)^{2} e^{-\gamma x} d x .
$$

Remark 3.1 The variable $x$ is as before interpreted as time to maturity. With the inner product

$$
(r, q)=\int_{0}^{\infty} r(x) q(x) e^{-a x} d x+\int_{0}^{\infty}\left(\frac{d r}{d x}(x)\right)\left(\frac{d q}{d x}(x)\right) e^{-\gamma x} d x,
$$

the space $\mathcal{H}_{\gamma}$ becomes a Hilbert space. Because of the exponential weighting function all constant forward rate curves will belong to the space. In the sequel we will suppress the subindex $\gamma$, writing $\mathcal{H}$ instead of $\mathcal{H}_{\gamma}$.

\subsubsection{The Forward Curve Manifold}

We consider as given a mapping

$$
G: \mathcal{Z} \rightarrow \mathcal{H}
$$

where the parameter space $\mathcal{Z}$ is an open connected subset of $R^{d}$, i.e. for each parameter value $z \in \mathcal{Z} \subseteq R^{d}$ we have a curve $G(z) \in \mathcal{H}$. The value of this curve at the point $x \in R_{+}$will be written as $G(z, x)$, so we see that $G$ can also be viewed as a mapping

$$
G: \mathcal{Z} \times R_{+} \rightarrow R
$$

The mapping $G$ is thus a formalization of the idea of a finitely parameterized family of forward rate curves, and we now define the forward curve manifold as the set of all forward rate curves produced by this family.

Definition 3.3 The forward curve manifold $\mathcal{G} \subseteq \mathcal{H}$ is defined as

$$
\mathcal{G}=\operatorname{Im}(G)
$$




\subsubsection{The Interest Rate Model}

We take as given a volatility function $\sigma$ of the form

$$
\sigma: \mathcal{H} \times R_{+} \rightarrow R^{m}
$$

i.e. $\sigma(r, x)$ is a functional of the infinite dimensional $r$-variable, and a function of the real variable $x$. Denoting the forward rate curve at time $t$ by $r_{t}$ we then have the following forward rate equation.

$$
d r_{t}(x)=\left\{\frac{\partial}{\partial x} r_{t}(x)+\sigma\left(r_{t}, x\right) \int_{0}^{x} \sigma\left(r_{t}, u\right)^{\star} d u\right\} d t+\sigma\left(r_{t}, x\right) d W_{t} .
$$

Remark 3.2 For notational simplicity we have assumed that the $r$-dynamics are time homogenous. The case when $\sigma$ is of the form $\sigma(t, r, x)$ can be treated in exactly the same way. See [3].

We need some regularity assumptions, and the main ones are as follows. See [3] for technical details.

Assumption 3.1 We assume the following .

- The volatility mapping $r \longmapsto \sigma(r)$ is smooth.

- The mapping $z \longmapsto G(z)$ is a smooth imbedding, so in particular the Frechet derivative $G_{z}^{\prime}(z)$ is injective for all $z \in \mathcal{Z}$.

- For every initial point $r_{0} \in \mathcal{G}$, there exists a unique strong solution in $\mathcal{H}$ of equation (40).

\subsubsection{The Problem}

Our main problem is the following.

- Suppose that we are given

- A volatility $\sigma$, specifying an interest rate model $\mathcal{M}$ as in (40)

- A mapping $G$, specifying a forward curve manifold $\mathcal{G}$.

- Is $\mathcal{G}$ then invariant under the action of $r$ ?

\subsection{The Invariance Conditions}

In order to study the invariance problem we need to introduce some compact notation. 
Definition 3.4 We define $\mathbf{H} \sigma$ by

$$
\mathbf{H} \sigma(r, x)=\int_{0}^{x} \sigma(r, s) d s
$$

Suppressing the $x$-variable, the Itô dynamics for the forward rates are thus given by

$$
d r_{t}=\left\{\frac{\partial}{\partial x} r_{t}+\sigma\left(r_{t}\right) \mathbf{H} \sigma\left(r_{t}\right)^{\star}\right\} d t+\sigma\left(r_{t}\right) d W_{t}
$$

and we write this more compactly as

$$
d r_{t}=\mu_{0}\left(r_{t}\right) d t+\sigma\left(r_{t}\right) d W_{t},
$$

where the drift $\mu_{0}$ is given by the bracket term in (41). To get some intuition we now formally "divide by $d t$ " and obtain

$$
\frac{d r}{d t}=\mu_{0}\left(r_{t}\right)+\sigma\left(r_{t}\right) \dot{W}_{t},
$$

where the formal time derivative $\dot{W}_{t}$ is interpreted as an "input signal" chosen by chance. As in Section 2.3 we are thus led to study the associated deterministic control system

$$
\frac{d r}{d t}=\mu_{0}\left(r_{t}\right)+\sigma\left(r_{t}\right) u_{t} .
$$

The intuitive idea is now that $\mathcal{G}$ is invariant under (42) if and only if $\mathcal{G}$ is invariant under (44) for all choices of the input signal $u$. It is furthermore geometrically obvious that this happens if and only if the velocity vector $\mu(r)+\sigma(r) u$ is tangential to $\mathcal{G}$ for all points $r \in \mathcal{G}$ and all choices of $u \in R^{m}$. Since the tangent space of $\mathcal{G}$ at a point $G(z)$ is given by $\operatorname{Im}\left[G_{z}^{\prime}(z)\right]$, where $G_{z}^{\prime}$ denotes the Frechet derivative (Jacobian), we are led to conjecture that $\mathcal{G}$ is invariant if and only if the condition

$$
\mu_{0}(r)+\sigma(r) u \in \operatorname{Im}\left[G_{z}^{\prime}(z)\right]
$$

is satisfied for all $u \in R^{m}$. This can also be written

$$
\begin{aligned}
\mu_{0}(r) & \in \operatorname{Im}\left[G_{z}^{\prime}(z)\right], \\
\sigma(r) & \in \operatorname{Im}\left[G_{z}^{\prime}(z)\right],
\end{aligned}
$$

where the last inclusion is interpreted componentwise for $\sigma$.

This "result" is, however, not correct due to the fact that the argument above neglects the difference between ordinary calculus, which is used for (44), and Itô calculus, which governs (42). In order to bridge this gap we have to rewrite the analysis in terms of Stratonovich integrals instead of Itô integrals.

Definition 3.5 For given semimartingales $X$ and $Y$, the Stratonovich integral of $X$ with respect to $Y, \int_{0}^{t} X(s) \circ d Y(s)$, is defined as

$$
\int_{0}^{t} X_{s} \circ d Y_{s}=\int_{0}^{t} X_{s} d Y_{s}+\frac{1}{2}\langle X, Y\rangle_{t}
$$


The first term on the RHS is the Itô integral. In the present case, with only Wiener processes as driving noise, we can define the 'quadratic variation process' $\langle X, Y\rangle$ in (45) by

$$
d\langle X, Y\rangle_{t}=d X_{t} d Y_{t},
$$

with the usual 'multiplication rules' $d W \cdot d t=d t \cdot d t=0, d W \cdot d W=d t$. We now recall the main result and raison d'être for the Stratonovich integral.

Proposition 3.1 (Chain rule) Assume that the function $F(t, y)$ is smooth. Then we have

$$
d F\left(t, Y_{t}\right)=\frac{\partial F}{\partial t}\left(t, Y_{t}\right) d t+\frac{\partial F}{\partial y} \circ d Y_{t} .
$$

Thus, in the Stratonovich calculus, the Itô formula takes the form of the standard chain rule of ordinary calculus.

Returning to (42), the Stratonovich dynamics are given by

$$
\begin{aligned}
d r_{t} & =\left\{\frac{\partial}{\partial x} r_{t}+\sigma\left(r_{t}\right) \mathbf{H} \sigma\left(r_{t}\right)^{\star}\right\} d t-\frac{1}{2} d\left\langle\sigma\left(r_{t}\right), W_{t}\right\rangle \\
& +\sigma\left(r_{t}\right) \circ d W_{t} .
\end{aligned}
$$

In order to compute the Stratonovich correction term above we use the infinite dimensional Itô formula (see [12]) to obtain

$$
d \sigma\left(r_{t}\right)=\{\ldots\} d t+\sigma_{r}^{\prime}\left(r_{t}\right) \sigma\left(r_{t}\right) d W_{t},
$$

where $\sigma_{r}^{\prime}$ denotes the Frechet derivative of $\sigma$ w.r.t. the infinite dimensional $r$ variable. From this we immediately obtain

$$
d\left\langle\sigma\left(r_{t}\right), W_{t}\right\rangle=\sigma_{r}^{\prime}\left(r_{t}\right) \sigma\left(r_{t}\right) d t .
$$

Remark 3.3 If the Wiener process $W$ is multidimensional, then $\sigma$ is a vector $\sigma=\left[\sigma_{1}, \ldots, \sigma_{m}\right]$, and the rhs of (50) should be interpreted as

$$
\sigma_{r}^{\prime}\left(r_{t}\right) \sigma\left(r_{t}, x\right)=\sum_{i=1}^{m} \sigma_{i r}^{\prime}\left(r_{t}\right) \sigma_{i}\left(r_{t}\right)
$$

Thus (48) becomes

$$
\begin{aligned}
d r_{t} & =\left\{\frac{\partial}{\partial x} r_{t}+\sigma\left(r_{t}\right) \mathbf{H} \sigma\left(r_{t}\right)^{\star}-\frac{1}{2} \sigma_{r}^{\prime}\left(r_{t}\right) \sigma\left(r_{t}\right)\right\} d t \\
& +\sigma\left(r_{t}\right) \circ d W_{t}
\end{aligned}
$$

We now write (51) as

$$
d r_{t}=\mu\left(r_{t}\right) d t+\sigma\left(r_{t}\right) \circ d W_{t}
$$


where

$$
\mu(r, x)=\frac{\partial}{\partial x} r(x)+\sigma\left(r_{t}, x\right) \int_{0}^{x} \sigma\left(r_{t}, u\right)^{\star} d u-\frac{1}{2}\left[\sigma_{r}^{\prime}\left(r_{t}\right) \sigma\left(r_{t}\right)\right](x) .
$$

Given the heuristics above, our main result is not surprising. The formal proof, which is somewhat technical, is left out. See [3].

Theorem 3.1 (Main Theorem) The forward curve manifold $\mathcal{G}$ is locally invariant for the forward rate process $r(t, x)$ in $\mathcal{M}$ if and only if,

$$
\begin{aligned}
G_{x}^{\prime}(z)+\sigma(r) \mathbf{H} \sigma(r)^{\star}-\frac{1}{2} \sigma_{r}^{\prime}(r) \sigma(r) & \in \operatorname{Im}\left[G_{z}^{\prime}(z)\right], \\
\sigma(r) & \in \operatorname{Im}\left[G_{z}^{\prime}(z)\right],
\end{aligned}
$$

hold for all $z \in \mathcal{Z}$ with $r=G(z)$.

Here, $G_{z}^{\prime}$ and $G_{x}^{\prime}$ denote the Frechet derivative of $G$ with respect to $z$ and $x$, respectively. The condition (55) is interpreted componentwise for $\sigma$. Condition (54) is called the consistent drift condition, and (55) is called the consistent volatility condition.

Remark 3.4 It is easily seen that if the family $G$ is invariant under shifts in the $x$-variable, then we will automatically have the relation

$$
G_{x}^{\prime}(z) \in \operatorname{Im}\left[G_{z}^{\prime}(z)\right]
$$

so in this case the relation (54) can be replaced by

$$
\sigma(r) \mathbf{H} \sigma(r)^{\star}-\frac{1}{2} \sigma_{r}^{\prime}(r) \sigma(r) \in \operatorname{Im}\left[G_{z}^{\prime}(z)\right],
$$

with $r=G(z)$ as usual.

\subsection{Examples}

The results above are extremely easy to apply in concrete situations. As a test case we consider the Nelson-Siegel (see [28]) family of forward rate curves. We analyze the consistency of this family with the Ho-Lee and Hull-White interest rate models. It should be emphasised that these examples are chosen only in

order to illustrate the general methodology. For more examples and details, see $[3]$.

\subsubsection{The Nelson-Siegel Family}

The Nelson-Siegel (henceforth NS) forward curve manifold $\mathcal{G}$ is parameterized by $z \in R^{4}$, the curve $x \longmapsto G(z, x)$ as

$$
G(z, x)=z_{1}+z_{2} e^{-z_{4} x}+z_{3} x e^{-z_{4} x} .
$$


For $z_{4} \neq 0$, the Frechet derivatives are easily obtained as

$$
\begin{gathered}
G_{z}^{\prime}(z, x)=\left[1, e^{-z_{4} x}, x e^{-z_{4} x},-\left(z_{2}+z_{3} x\right) x e^{-z_{4} x}\right], \\
G_{x}^{\prime}(z, x)=\left(z_{3}-z_{2} z_{4}-z_{3} z_{4} x\right) e^{-z_{4} x} .
\end{gathered}
$$

In order for the image of this map to be included in $\mathcal{H}_{\gamma}$, we need to impose the condition $z_{4}>-\gamma / 2$. In this case, the natural parameter space is thus $\mathcal{Z}=\left\{z \in R^{4}: z_{4} \neq 0, z_{4}>-\gamma / 2\right\}$. However, as we shall see below, the results are uniform w.r.t. $\gamma$. Note that the mapping $G$ indeed is smooth, and for $z_{4} \neq 0$, $G$ and $G_{z}^{\prime}$ are also injective.

In the degenerate case $z_{4}=0$, we have

$$
G(z, x)=z_{1}+z_{2}+z_{3} x
$$

We return to this case below.

\subsubsection{The Hull-White and Ho-Lee Models}

As our test case, we analyze the Hull and White (henceforth HW) extension of the Vasiček model. On short rate form the model is given by

$$
d R(t)=\{\Phi(t)-a R(t)\} d t+\sigma d W(t),
$$

where $a, \sigma>0$. As is well known, the corresponding forward rate formulation is

$$
d r(t, x)=\beta(t, x) d t+\sigma e^{-a x} d W_{t} .
$$

Thus, the volatility function is given by $\sigma(x)=\sigma e^{-a x}$, and the conditions of Theorem 3.1 become

$$
\begin{aligned}
G_{x}^{\prime}(z, x)+\frac{\sigma^{2}}{a}\left[e^{-a x}-e^{-2 a x}\right] & \in \operatorname{Im}\left[G_{z}^{\prime}(z, x)\right], \\
\sigma e^{-a x} & \in \operatorname{Im}\left[G_{z}^{\prime}(z, x)\right] .
\end{aligned}
$$

To investigate whether the NS manifold is invariant under HW dynamics, we start with (63) and fix a $z$-vector. We then look for constants (possibly depending on $z) A, B, C$, and $D$, such that for all $x \geq 0$ we have

$$
\sigma e^{-a x}=A+B e^{-z_{4} x}+C x e^{-z_{4} x}-D\left(z_{2}+z_{3} x\right) x e^{-z_{4} x} .
$$

This is possible if and only if $z_{4}=a$, and since (63) must hold for all choices of $z \in \mathcal{Z}$ we immediately see that HW is inconsistent with the full NS manifold (see also the Notes below).

Proposition 3.2 (Nelson-Siegel and Hull-White) The Hull-White model is inconsistent with the NS family. 
We have thus obtained a negative result for the HW model. The NS manifold is 'too small' for HW, in the sense that if the initial forward rate curve is on the manifold, then the HW dynamics will force the term structure off the manifold within an arbitrarily short period of time. For more positive results see [3].

Remark 3.5 It is an easy exercise to see that the minimal manifold which is consistent with HW is given by

$$
G(z, x)=z_{1} e^{-a x}+z_{2} e^{-2 a x} .
$$

In the same way, one may easily test the consistency between NS and the model obtained by setting $a=0$ in (60). This is the continuous time limit of the Ho and Lee model [21], and is henceforth referred to as HL. Since we have a pedagogical point to make, we give the results on consistency, which are as follows.

\section{Proposition 3.3 (Nelson-Siegel and Ho-Lee)}

(a) The full NS family is inconsistent with the Ho-Lee model.

(b) The degenerate family $G(z, x)=z_{1}+z_{3} x$ is in fact consistent with Ho-Lee.

Remark 3.6 We see that the minimal invariant manifold provides information about the model. From the result above, the HL model is closely tied to the class of affine forward rate curves. Such curves are unrealistic from an economic point of view, implying that the HL model is overly simplistic.

\subsection{Notes}

The section is based on [3]. As we very easily detected above, neither the HW nor the HL model is consistent with the Nelson-Siegel famliy of forward rate curves. A much more difficult problem is to determine whether any interest rate model is. This is Problem II in Section 3.1 for the NS family, and it has been solved recently (using different techniques) in [17], where it is shown that no nontrivial Wiener driven model is consistent with NS. Thus, for a model to be consistent with Nelson-Siegel, it must be deterministic. In [18] (which is a technical tour de force) this result is extended to a much larger exponential polynomial family than the NS family. In our presentation we have used strong solutions of the infinite dimensional forward rate SDE. This is of course restrictive. The invariance problem for weak solutions has recently been studied in [19]. An alternative way of studying invariance is by using some version of the Stroock-Varadhan support theorem, and this line of thought is carried out in depth in [32]. 


\section{Existence of Nonlinear Realizations}

We now turn to Problem 2 in Section 1.2, i.e. the problem when a given forward rate model has a finite dimensional factor realization. For ease of exposition we mostly confine ourselves to a discussion of the case of a single driving Wiener process and to time invariant forward rate dynamics. Multidimensional Wiener processes, and time varying systems can be treated similarly, and for completeness we state the results for the multidimensional case. We will use some ideas and concepts from differential geometry, and a general reference here is [30]. The section is based on [5].

\subsection{Setup}

In order to study the realization problem we need (see Remark 4.1) a very regular space to work in.

Definition 4.1 Consider a fixed real number $\gamma>0$. The space $\mathcal{B}_{\gamma}$ is defined as the space of all infinitely differentiable functions

$$
r: R_{+} \rightarrow R
$$

satisfying the norm condition $\|r\|_{\gamma}<\infty$. Here the norm is defined as

$$
\|r\|_{\gamma}^{2}=\sum_{n=0}^{\infty} 2^{-n} \int_{0}^{\infty}\left(\frac{d^{n} r}{d x^{n}}(x)\right)^{2} e^{-\gamma x} d x .
$$

Note that $\mathcal{B}$ is not a space of distributions, but a space of functions. As with $\mathcal{H}$ we will often suppress the subindex $\gamma$. With the obvious inner product $\mathcal{B}$ is a pre-Hilbert space, and in [5] the following result is proved.

Proposition 4.1 The space $\mathcal{B}$ is a Hilbert space, i.e. it is complete. Furthermore, every function in the space is fact real analytic, and can thus be uniquely extended to a holomorphic function in the entire complex plane.

We now take as given a volatility $\sigma: \mathcal{B} \rightarrow B$ and consider the induced forward rate model (on Stratonovich form)

$$
d r_{t}=\mu\left(r_{t}\right) d t+\sigma\left(r_{t}\right) \circ d W_{t}
$$

where as before (see Section 3.4).

$$
\mu(r)=\frac{\partial}{\partial x} r+\sigma(r) \mathbf{H} \sigma(r)^{\star}-\frac{1}{2} \sigma_{r}^{\prime}(r) \sigma(r)
$$

We need some regularity assumptions. 
Assumption 4.1 We assume that $\sigma$ is chosen such that the following hold.

- The mapping $\sigma$ is smooth.

- The mapping

$$
r \longmapsto \sigma(r) \mathbf{H} \sigma(r)^{\star}-\frac{1}{2} \sigma_{r}^{\prime}(r) \sigma(r)
$$

is a smooth map from $\mathcal{B}$ to $\mathcal{B}$.

Remark 4.1 The reason for our choice of $\mathcal{B}$ as the underlying space, is that the linear operator $\mathbf{F}=d / d x$ is bounded in this space. Together with the assumptions above, this implies that both $\mu$ and $\sigma$ are smooth vector fields on $\mathcal{B}$, thus ensuring the existence of a strong local solution to the forward rate equation for every initial point $r^{o} \in \mathcal{B}$.

\subsection{The Geometric problem}

Given a specification of the volatility mapping $\sigma$, and an initial forward rate curve $r^{o}$ we now investigate when (and how) the corresponding forward rate process possesses a finite, dimensional realization. We are thus looking for smooth $d$-dimensional vector fields $a$ and $b$, an initial point $z_{0} \in R^{d}$, and a mapping $G: R^{d} \rightarrow \mathcal{B}$ such that $r$, locally in time, has the representation

$$
\begin{aligned}
d Z_{t} & =a\left(Z_{t}\right) d t+b\left(Z_{t}\right) d W_{t}, Z_{0}=z_{0} \\
r(t, x) & =G\left(Z_{t}, x\right) .
\end{aligned}
$$

Remark 4.2 Let us clarify some points. Firstly, note that in principle it may well happen that, given a specification of $\sigma$, the $r$-model has a finite dimensional realization given a particular initial forward rate curve $r^{o}$, while being infinite dimensional for all other initial forward rate curves in a neighbourhood of $r^{o}$. We say that such a model is a non-generic or accidental finite dimensional model. If, on the other hand, $r$ has a finite dimensional realization for all initial points in a neighbourhood of $r^{o}$, then we say that the model is a generically finite dimensional model. In this text we are solely concerned with the generic problem. Secondly, let us emphasise that we are looking for local (in time) realizations.

We can now connect the realization problem to our studies of invariant manifolds.

Proposition 4.2 The forward rate process possesses a finite dimensional realization if and only if there exists an invariant finite dimensional submanifold $\mathcal{G}$ with $r^{o} \in \mathcal{G}$. 
Proof. See [3] for the full proof. The intuitive argument runs as follows. Suppose that there exists a finite dimensional invariant manifold $\mathcal{G}$ with $r^{o} \in \mathcal{G}$. Then $\mathcal{G}$ has a local coordinate system, and we may define the $Z$ process as the local coordinate process for the $r$-process. On the other hand it is clear that if $r$ has a finite dimensional realization as in (67)-(68), then every forward rate curve that will be produced by the model is of the form $x \longmapsto G(z, x)$ for some choice of $z$. Thus there exists a finite dimensional invariant submanifold $\mathcal{G}$ containing the initial forward rate curve $r^{o}$, namely $\mathcal{G}=\operatorname{Im} G$.

Using Theorem 3.1 we immediately obtain the following geometric characterisation of the existence of a finite realization.

Corollary 4.1 The forward rate process possesses a finite dimensional realization if and only if there exists a finite dimensional manifold $\mathcal{G}$ containing $r^{o}$, such that, for each $r \in \mathcal{G}$ the following conditions hold.

$$
\begin{aligned}
& \mu(r) \in T_{\mathcal{G}}(r), \\
& \sigma(r) \in T_{\mathcal{G}}(r) .
\end{aligned}
$$

Here $T_{\mathcal{G}}(r)$ denotes the tangent space to $\mathcal{G}$ at the point $r$, and the vector fields $\mu$ and $\sigma$ are as above.

\subsection{The Main Result}

Given the volatility vector field $\sigma$, and hence also the field $\mu$, we now are faced with the problem of determining if there exists a finite dimensional manifold $\mathcal{G}$ with the property that $\mu$ and $\sigma$ are tangential to $\mathcal{G}$ at each point of $\mathcal{G}$. In the case when the underlying space is finite dimensional, this is a standard problem in differential geometry, and we will now give the heuristics.

To get some intuition we start with a simpler problem and therefore consider the space $\mathcal{B}$ (or any other Hilbert space), and a smooth vector field $f$ on the space. For each fixed point $r^{o} \in \mathcal{B}$ we now ask if there exists a finite dimensional manifold $\mathcal{G}$ with $r^{o} \in \mathcal{G}$ such that $f$ is tangential to $\mathcal{G}$ at every point. The answer to this question is yes, and the manifold can in fact be chosen to be one-dimensional. To see this, consider the infinite dimensional ODE

$$
\begin{aligned}
\frac{d r_{t}}{d t} & =f\left(r_{t}\right), \\
r_{0} & =r^{o} .
\end{aligned}
$$

If $r_{t}$ is the solution, at time $t$, of this ODE, we use the notation

$$
r_{t}=e^{f t} r^{o} .
$$

We have thus defined a group of operators $\left\{e^{f t}: t \in R\right\}$, and we note that the set $\left\{e^{f t} r^{o}: t \in R\right\} \subseteq \mathcal{B}$ is nothing else than the integral curve of the vector field 
$f$, passing through $r^{o}$. If we define $\mathcal{G}$ as this integral curve, then our problem is solved, since $f$ will be tangential to $\mathcal{G}$ by construction.

Let us now take two vector fields $f_{1}$ and $f_{2}$ as given, where the reader informally can think of $f_{1}$ as $\sigma$ and $f_{2}$ as $\mu$. We also fix an initial point $r^{o} \in \mathcal{B}$ and the question is if there exists a finite dimensional manifold $\mathcal{G}$, containing $r^{o}$, with the property that $f_{1}$ and $f_{2}$ are both tangential to $\mathcal{G}$ at each point of $\mathcal{G}$. We call such a manifold an tangential manifold for the vector fields. At a first glance it would seem that there always exists an tangential manifold, and that it can even be chosen to be two-dimensional. The geometric idea is that we start at $r^{o}$ and let $f_{1}$ generate the integral curve $\left\{e^{f_{1} s} r^{o}: s \geq 0\right\}$. For each point $e^{f_{1} s} r^{o}$ on this curve we now let $f_{2}$ generate the integral curve starting at that point. This gives us the object $e^{f_{2} t} e^{f_{1} s} r^{o}$ and thus it seems that we sweep out a two dimensional surface $\mathcal{G}$ in $\mathcal{B}$. This is our obvious candidate for an tangential manifold.

In the general case this idea will, however, not work, and the basic problem is as follows. In the construction above we started with the integral curve generated by $f_{1}$ and then applied $f_{2}$, and there is of course no guarantee that we will obtain the same surface if we start with $f_{2}$ and then apply $f_{1}$. We thus have some sort of commutativity problem, and the key concept is the Lie bracket.

Definition 4.2 Given smooth vector fields $f$ and $g$ on $\mathcal{B}$, the Lie bracket $[f, g]$ is a new vector field defined by

$$
[f, g](r)=f^{\prime}(r) g(r)-g^{\prime}(r) f(r)
$$

The Lie bracket measures the lack of commutativity on the infinitesimal scale in our geometric program above, and for the procedure to work we need a condition which says that the lack of commutativity is "small". It turns out that the relevant condition is that the Lie bracket should be in the linear hull of the vector fields.

Definition 4.3 Let $f_{1}, \ldots, f_{n}$ be smooth independent vector fields on some space $X$. Such a system is called a distribution, and the distribution is said to be involutive if

$$
\left[f_{i}, f_{j}\right](x) \in \operatorname{span}\left\{f_{1}(x), \ldots, f_{n}(x)\right\}, \quad \forall i, j,
$$

where the span is the linear hull over the real numbers.

We now have the following basic result, which extends a classic result from finite dimensional differential geometry (see [30]).

Theorem 4.1 (Frobenius) Let $f_{1}, \ldots, f_{k}$ and be independent smooth vector fields in $\mathcal{B}$ and consider a fixed point $r^{o} \in \mathcal{B}$. Then the following statements are equivalent. 
- For each point $r$ in a neighbourhood of $r^{o}$, there exists a $k$-dimensional tangential manifold passing through $r$.

- The system $f_{1}, \ldots, f_{k}$ of vector fields is (locally) involutive.

Proof. See [5], which provides a self contained proof of the Frobenius Theorem in Banach space.

Let us now go back to our interest rate model. We are thus given the vector fields $\mu, \sigma$, and an initial point $r^{o}$, and the problem is whether there exists a finite dimensional tangential manifold containing $r^{o}$. Using the infinite dimensional Frobenius theorem, this situation is now easily analyzed. If $\{\mu, \sigma\}$ is involutive then there exists a two dimensional tangential manifold. If $\{\mu, \sigma\}$ is not involutive, this means that the Lie bracket $[\mu, \sigma]$ is not in the linear span of $\mu$ and $\sigma$, so then we consider the system $\{\mu, \sigma,[\mu, \sigma]\}$. If this system is involutive there exists a three dimensional tangential manifold. If it is not involutive at least one of the brackets $[\mu,[\mu, \sigma]],[\sigma,[\mu, \sigma]]$ is not in the span of $\{\mu, \sigma,[\mu, \sigma]\}$, and we then adjoin this (these) bracket(s). We continue in this way, forming brackets of brackets, and adjoining these to the linear hull of the previously obtained vector fields, until the point when the system of vector fields thus obtained actually is closed under the Lie bracket operation.

Definition 4.4 Take the vector fields $f_{1}, \ldots, f_{k}$ as given. The Lie algebra generated by $f_{1}, \ldots, f_{k}$ is the smallest linear space (over $R$ ) of vector fields which contains $f_{1}, \ldots, f_{k}$ and is closed under the Lie bracket. This Lie algebra is denoted by

$$
\mathcal{L}=\left\{f_{1}, \ldots, f_{k}\right\}_{L A}
$$

The dimension of $\mathcal{L}$ is defined, for each point $r \in \mathcal{B}$ as

$$
\operatorname{dim}[\mathcal{L}(r)]=\operatorname{dim} \operatorname{span}\left\{f_{1}(r), \ldots, f_{k}(r)\right\} .
$$

Putting all these results together, we have the following main result on finite dimensional realizations.

Theorem 4.2 (Main Result) Take the volatility mapping $\sigma=\left(\sigma_{1}, \ldots, \sigma_{m}\right)$ as given. Then the forward rate model generated by $\sigma$ generically admits a finite dimensional realization if and only if

$$
\operatorname{dim}\left\{\mu, \sigma_{1}, \ldots, \sigma_{m}\right\}_{L A}<\infty
$$

in a neighbourhood of $r^{o}$.

The result above thus provides a general solution to Problem II from Section 1.2. For any given specification of forward rate volatilities, the Lie algebra can 
in principle be computed, and the dimension can be checked. Note, however, that the theorem is a pure existence result. If, for example, the Lie algebra has dimension five, then we know that there exists a five-dimensional realisation, but the theorem does not directly tell us how to construct a concrete realization. This is the subject of ongoing research. Note also that realizations are not unique, since any diffeomorphic mapping of the factor space $R^{d}$ onto itself will give a new equivalent realization.

When computing the Lie algebra generated by $\mu$ and $\sigma$, the following observations are often useful.

Lemma 4.1 Take the vector fields $f_{1}, \ldots, f_{k}$ as given. The Lie algebra $\mathcal{L}=$ $\left\{f_{1}, \ldots, f_{k}\right\}_{L A}$ remains unchanged under the following operations.

- The vector field $f_{i}(r)$ may be replaced by $\alpha(r) f_{i}(r)$, where $\alpha$ is any smooth nonzero scalar field.

- The vector field $f_{i}(r)$ may be replaced by

$$
f_{i}(r)+\sum_{j \neq i} \alpha_{j}(r) f_{j}(r)
$$

where $\alpha_{j}$ is any smooth scalar field.

Proof. The first point is geometrically obvious, since multiplication by a scalar field will only change the length of the vector field $f_{i}$, and not its direction, and thus not the tangential manifold. Formally it follows from the "Leibnitz rule" $[f, \alpha g]=\alpha[f, g]-\left(\alpha^{\prime} f\right) g$. The second point follows from the bilinear property of the Lie bracket together with the fact that $[f, f]=0$.

\subsection{Applications}

In this section we give some simple applications of the theory developed above. For more examples and results, see [5].

\subsubsection{Constant Volatility}

We start with the simplest case, which is when the volatility $\sigma(r, x)$ is a constant vector in $\mathcal{B}$. We are thus back in the framework of Section 2 , and we assume for simplicity that we have only one driving Wiener process. Then we have no Stratonovich correction term and the vector fields are given by

$$
\begin{aligned}
\mu(r, x) & =\mathbf{F} r(x)+\sigma(x) \int_{0}^{x} \sigma(s) d s, \\
\sigma(r, x) & =\sigma(x) .
\end{aligned}
$$


where as before $\mathbf{F}=\frac{\partial}{\partial x}$.

The Frechet derivatives are trivial in this case. Since $\mathbf{F}$ is linear (and bounded in our space), and $\sigma$ is constant as a function of $r$, we obtain

$$
\begin{aligned}
& \mu_{r}^{\prime}=\mathbf{F}, \\
& \sigma_{r}^{\prime}=0 .
\end{aligned}
$$

Thus the Lie bracket $[\mu, \sigma]$ is given by

$$
[\mu, \sigma]=\mathbf{F} \sigma,
$$

and in the same way we have

$$
[\mu,[\mu, \sigma]]=\mathbf{F}^{2} \sigma .
$$

Continuing in the same manner it is easily seen that the relevant Lie algebra $\mathcal{L}$ is given by

$$
\mathcal{L}=\{\mu, \sigma\}_{L A}=\operatorname{span}\left\{\mu, \sigma, \mathbf{F} \sigma, \mathbf{F}^{2} \sigma, \ldots\right\}=\operatorname{span}\left\{\mu, \mathbf{F}^{n} \sigma ; n=0,1,2, \ldots\right\}
$$

It is thus clear that $\mathcal{L}$ is finite dimensional (at each point $r$ ) if and only if the function space

$$
\operatorname{span}\left\{\mathbf{F}^{n} \sigma ; n=0,1,2, \ldots\right\}
$$

is finite dimensional. We have thus obtained our old condition from Proposition 2.3 and we have the following result which extends Proposition 2.2 by in principle allowing the realization to be non-linear.

Proposition 4.3 Under the above assumptions, there exists a finite dimensional realization if and only if $\sigma$ is a quasi-exponential function.

\subsubsection{Constant Direction Volatility}

We go on to study the most natural extension of the deterministic volatility case (still in the case of a scalar Wiener process) namely the case when the volatility is of the form

$$
\sigma(r, x)=\varphi(r) \lambda(x) .
$$

In this case the individual vector field $\sigma$ has the constant direction $\lambda \in \mathcal{H}$, but is of varying length, determined by $\varphi$, where $\varphi$ is allowed to be any smooth functional of the entire forward rate curve. In order to avoid trivialities we make the following assumption.

Assumption 4.2 We assume that $\varphi(r) \neq 0$ for all $r \in \mathcal{H}$.

After a simple calculation the drift vector $\mu$ turns out to be

$$
\mu(r)=\mathbf{F} r+\varphi^{2}(r) D-\frac{1}{2} \varphi^{\prime}(r)[\lambda] \varphi(r) \lambda,
$$


where $\varphi^{\prime}(r)[\lambda]$ denotes the Frechet derivative $\varphi^{\prime}(r)$ acting on the vector $\lambda$, and where the constant vector $D \in \mathcal{H}$ is given by

$$
D(x)=\lambda(x) \int_{0}^{x} \lambda(s) d s .
$$

We now want to know under what conditions on $\varphi$ and $\lambda$ we have a finite dimensional realization, i.e. when the Lie algebra generated by

$$
\begin{aligned}
& \mu(r)=\mathbf{F} r+\varphi^{2}(r) D-\frac{1}{2} \varphi^{\prime}(r)[\lambda] \varphi(r) \lambda, \\
& \sigma(r)=\varphi(r) \lambda,
\end{aligned}
$$

is finite dimensional. Under Assumption 4.2 we can use Lemma 4.1, to see that the Lie algebra is in fact generated by the simpler system of vector fields

$$
\begin{aligned}
& f_{0}(r)=\mathbf{F} r+\Phi(r) D, \\
& f_{1}(r)=\lambda
\end{aligned}
$$

where we have used the notation

$$
\Phi(r)=\varphi^{2}(r) .
$$

Since the field $f_{1}$ is constant, it has zero Frechet derivative. Thus the first Lie bracket is easily computed as

$$
\left[f_{0}, f_{1}\right](r)=\mathbf{F} \lambda+\Phi^{\prime}(r)[\lambda] D .
$$

The next bracket to compute is $\left[\left[f_{0}, f_{1}\right], f_{1}\right]$ which is given by

$$
\left[\left[f_{0}, f_{1}\right], f_{1}\right]=\Phi^{\prime \prime}(r)[\lambda ; \lambda] D .
$$

Note that $\Phi^{\prime \prime}(r)[\lambda ; \lambda]$ is the second order Frechet derivative of $\Phi$ operating on the vector pair $[\lambda ; \lambda]$. This pair is to be distinguished from (notice the semicolon) the Lie bracket $[\lambda, \lambda]$ (with a comma), which if course would be equal to zero. We now make a further assumption.

Assumption 4.3 We assume that $\Phi^{\prime \prime}(r)[\lambda ; \lambda] \neq 0$ for all $r \in \mathcal{H}$.

Given this assumption we may again use Lemma 4.1 to see that the Lie algebra is generated by the following vector fields

$$
\begin{aligned}
& f_{0}(r)=\mathbf{F} r \\
& f_{1}(r)=\lambda, \\
& f_{3}(r)=\mathbf{F} \lambda, \\
& f_{4}(r)=D .
\end{aligned}
$$


Of these vector fields, all but $f_{0}$ are constant, so all brackets are easy. After elementary calculations we see that in fact

$$
\{\mu, \sigma\}_{L A}=\operatorname{span}\left\{\mathbf{F} r, \mathbf{F}^{n} \lambda, \mathbf{F}^{n} D ; n=0,1, \ldots\right\} .
$$

From this expression it follows immediately that a necessary condition for the Lie algebra to be finite dimensional is that the vector space spanned by $\left\{\mathbf{F}^{n} \lambda ; n \geq 0\right\}$ is finite dimensional. This occurs if and only if $\lambda$ is quasi-exponential (see Remark 2.1). If, on the other hand, $\lambda$ is quasi-exponential, then we know from Lemma 2.1, that also $D$ is quasi-exponential, since it is the integral of the $\mathrm{QE}$ function $\lambda$ multiplied by the QE function $\lambda$. Thus the space $\left\{\mathbf{F}^{n} D ; n=0,1, \ldots\right\}$ is also finite dimensional, and we have proved the following result.

Proposition 4.4 Under Assumptions 4.2 and 4.3, the interest rate model with volatility given by $\sigma(r, x)=\varphi(r) \lambda(x)$ has a finite dimensional realization if and only if $\lambda$ is a quasi-exponential function. The scalar field $\varphi$ is allowed to be any smooth field.

\subsubsection{When is the Short Rate a Markov Process?}

One of the classical problems concerning the HJM approach to interest rate modelling is that of determining when a given forward rate model is realized by a short rate model, i.e. when the short rate is Markovian. We now briefly indicate how the theory developed above can be used in order to analyze this question. For the full theory see [5].

Using the results above, we immediately have the following general necessary condition.

Proposition 4.5 The forward rate model generated by $\sigma$ is a generic short rate model, i.e the short rate is generically a Markov process, only if

$$
\operatorname{dim}\{\mu, \sigma\}_{L A} \leq 2
$$

Proof. If the model is really a short rate model, then bond prices are given as $p(t, x)=F\left(t, R_{t}, x\right)$ where $F$ solves the term structure PDE. Thus bond prices, and forward rates are generated by a two dimensional factor model with time $t$ and the short rate $R$ as the state variables.

Remark 4.3 The most natural case is $\operatorname{dim}\{\mu, \sigma\}_{L A}=2$. It is an open problem whether there exists a non-deterministic generic short rate model with $\operatorname{dim}\{\mu, \sigma\}_{L A}=1$. 
Note that condition (74) is only a sufficient condition for the existence of a short rate realization. It guarantees that there exists a two-dimensional realization, but the question remains whether the realization can chosen in such a way that the short rate and running time are the state variables. This question is completely resolved by the following central result.

Theorem 4.3 Assume that the model is not deterministic, and take as given a time invariant volatility $\sigma(r, x)$. Then there exists a short rate realization if and only if the vector fields $[\mu, \sigma]$ and $\sigma$ are parallel, i.e. if and only if there exists a scalar field $\alpha(r)$ such that the following relation holds (locally) for all $r$.

$$
[\mu, \sigma](r)=\alpha(r) \sigma(r) .
$$

Proof. See [5].

It turns out that the class of generic short rate models is very small indeed. We have, in fact, the following result, which was first proved in [25] (using techniques different from those above). See [5] for a proof based on Theorem 4.3.

Theorem 4.4 Consider a HJM model with one driving Wiener process and a volatility structure of the form

$$
\sigma(r, x)=g(R, x) .
$$

where $R=r(0)$ is the short rate. Then the model is a generic short rate model if and only if $g$ has one of the following forms.

- There exists a constant $c$ such that

$$
g(R, x) \equiv c .
$$

- There exist constants a and $c$ such that.

$$
g(R, x)=c e^{-a x} .
$$

- There exist constants $a$ and $b$, and a function $\alpha(x)$, where $\alpha$ satisfies a certain Riccati equation, such that

$$
g(R, x)=\alpha(x) \sqrt{a R+b}
$$

We immediately recognize these cases as the Ho-Lee model, the Hull-White extended Vasiček model, and the Hull-White extended Cox-Ingersoll-Ross model. Thus, in this sense the only generic short rate models are the affine ones, and the moral of this, perhaps somewhat surprising, result is that most short rate models considered in the literature are not generic but "accidental". To understand the geometric picture one can think of the following program. 
1. Choose an arbitrary short rate model, say of the form

$$
d R_{t}=a\left(R_{t}\right) d t+b\left(R_{t}\right) d W_{t}
$$

with a fixed initial point $R_{0}$.

2. Solve the associated PDE in order to compute bond prices. This will also produce:

- An initial forward rate curve $\hat{r}^{o}(x)$.

- Forward rate volatilities of the form $g(R, x)$.

3. Forget about the underlying short rate model, and take the forward rate volatility structure $g(R, x)$ as given in the forward rate equation.

4. Initiate the forward rate equation with an arbitrary initial forward rate curve $r^{o}(x)$

The question is now whether the thus constructed forward rate model will produce a Markovian short rate process. Obviously, if you choose the initial forward rate curve $r^{o}$ as $r^{o}=\hat{r}^{o}$, then you are back where you started, and everything is OK. If, however, you choose another initial forward rate curve than $\hat{r}^{o}$, say the observed forward rate curve of today, then it is no longer clear that the short rate will be Markovian. What the theorem above says, is that only the models listed above will produce a Markovian short rate model for all initial points in a neighbourhood of $\hat{r}^{o}$. If you take another model (like, say, the Dothan model) then a generic choice of the initial forward rate curve will produce a short rate process which is not Markovian.

\subsection{Notes}

The section is based on [5] where full proofs and further results can be found, and where also the time varying case is considered. In our study of the constant direction model above, $\varphi$ was allowed to be any smooth functional of the entire forward rate curve. The simpler special case when $\varphi$ is a point evaluation of the short rate, i.e. of the form $\varphi(r)=h(r(0))$ has been studied in [1], [23] and [29].

All these cases falls within our present framwork and, the results are included as special cases of the general theory above. A different case, treated in [10], occurs when $\sigma$ is a finite point evaluation, i.e. when $\sigma(t, r)=h\left(t, r\left(x_{1}\right), \ldots r\left(x_{k}\right)\right)$ for fixed benchmark maturities $x_{1}, \ldots, x_{k}$. In [10] it is studied when the corresponding finite set of benchmark forward rates is Markovian.

A classic paper on Markovian short rates is [9], where a deterministic volatility of the form $\sigma(t, x)$ is considered. Theorem 4.4 was first stated and proved in [25]. See [14] for an example with a driving Levy process.

The geometric ideas presented above and in [5] are intimately connected to controllability problems in systems theory, where they have been used extensively 
(see [24]). They have also been used in filtering theory, where the problem is to find a finite dimensional realization of the unnormalized conditional density

process, the evolution of which is given by the Zakai equation. See [8] for an overview of these areas.

\section{References}

[1] Bhar, R. \& Chiarella, C. (1997) Transformation of Heath-Jarrow-Morton models to markovian systems. European Journal of Finance, 3, No. 1, 1-26.

[2] Björk, T. (1997). Interest Rate Theory. In W. Runggaldier (ed.), Financial Mathematics. Springer Lecture Notes in Mathematics, Vol. 1656. Springer Verlag, Berlin.

[3] Björk, T. \& Christensen, B.J. (1999) Interest rate dynamics and consistent forward rate curves. Mathematical Finance, 9, No. 4, 323-348.

[4] Björk, T. \& Gombani A. (1999) Minimal realization of interest rate models. Finance and Stochastics, 3, No. 4, 413-432.

[5] Björk, T. \& Svensson, L. (1999) On the existence of finite dimensional nonlinear realizations of interest rate models. Working paper, Stockholm School of Economics.

[6] Brace, A. \& Musiela M. (1994) A multi factor Gauss Markov implementation of Heath Jarrow and Morton. Mathematical Finance 4, Vol.3, 563-576.

[7] Brockett, R.W. (1970) Finite Dimensional Linear Systems. Wiley.

[8] Brockett, R.W. (1981) Nonlinear systems and nonlinear estimation theory. In Stochastic systems: The mathematics of filtering and identification and applications (eds. Hazewinkel, M \& Willems, J.C.) Reidel.

[9] Carverhill, A. (1994) When is the spot rate Markovian? Mathematical Finance, 4, 305-312.

[10] Chiarella, C \& Kwon, K. (1998) Forward rate dependent Markovian transformations of the Heath-Jarrow-Morton term structure model. Working paper. School of Finance and Economics, University of Technology, Sydney.

[11] Cox, J., Ingersoll, J. \& Ross, S. (1985b). A Theory of the Term Structure of Interest Rates. Econometrica 53, 385-408.

[12] Da Prato, G. \& Zabczyk, J.(1992) Stochastic Equations in Infinite Dimensions. Cambridge University Press.

[13] Duffie, D. \& Kan, R (1996) A yield factor model of interest rates. Mathematical Finance, 6, 379-406. 
[14] Eberlein, E. \& Raible, S. (1999) Term structure models driven by general Levy processes. Mathematical Finance, 9, 31-53.

[15] El Karoui, N. \& Lacoste, V (1993) Multifactor models of the term structure of interest rates . Preprint

[16] El Karoui, N. \& Geman, H. \& Lacoste, V (1997) On the role of state variables in interest rate models. Preprint

[17] Filipović, D. (1998a): A Note on the Nelson-Siegel family. Mathematical Finance, 9, No. 4, 349-359.

[18] Filipović, D. (1998b): Exponential-polynomial families and the term structure of interest rates. To appear in Bernoulli.

[19] Filipović, D. (1999): Invariant manifolds for weak solutions of stochastic equations. To appear in Probability Theory and Related Fields.

[20] Heath, D. \& Jarrow, R. \& Morton, A. (1992) Bond pricing and the term structure of interest rates. Econometrica 60 No.1, 77-106.

[21] Ho, T. \& Lee, S. (1986) Term structure movements and pricing interest rate contingent claims. Journal of Finance 41, 1011-1029.

[22] Hull, J \& White, A. (1990) Pricing interest-rate-derivative securities. The Review of Financial Studies 3, 573-592.

[23] Inui, K. \& Kijima, M. (1998) A markovian framework in multi-factor HeathJarrow-Morton models. JFQA 333 no. 3, 423-440.

[24] Isidori, A. (1989) Nonlinear Control Systems. Springer-Verlag, Berlin.

[25] Jeffrey, A. (1995) Single factor Heath-Jarrow-Morton term structure models based on Markovian spot interest rates. JFQA 30 no.4, 619-642.

[26] Musiela M. (1993) Stochastic PDE:s and term structure models. Preprint.

[27] Musiela, M. \& Rutkowski, M. (1997). Martingale Methods in Financial Modeling. Springer Verlag, Berlin Heidelberg New York.

[28] Nelson, C. \& Siegel, A. (1987): Parsimonious modelling of yield curves. Journal of Business, 60, 473-489.

[29] Ritchken, P. \& Sankarasubramanian, L. (1995) Volatility structures of forward rates and the dynamics of the term structure. mathematical Finance, 5, no. 1, 55-72.

[30] Warner, F.W. (1979) Foundations of differentiable manifolds and Lie groups. Scott, Foresman, Hill.

[31] Vasiček, O. (1977) An equilibrium characterization of the term structure. Journal of Financial Economics 5, 177-188. 
[32] Zabczyk, J.(1992) Stochastic invariance and conistency of financial models. Preprint. Scuola Normale Superiore, Pisa. 